\title{
BMJ Open Sleep and daytime problems during the COVID-19 pandemic and effects of coronavirus infection, confinement and financial suffering: a multinational survey using a harmonised questionnaire
}

Markku Partinen (D) , ${ }^{1,2}$ Brigitte Holzinger, ${ }^{3}$ Charles M Morin, ${ }^{4}$ Colin Espie, ${ }^{5}$ Frances Chung (D) , ${ }^{6}$ Thomas Penzel (D) , ${ }^{7}$ Christian Benedict, ${ }^{8}$ Courtney J Bolstad, ${ }^{9}$ Jonathan Cedernaes, ${ }^{10,11}$ Rachel Ngan Yin Chan, ${ }^{12}$ Yves Dauvilliers, ${ }^{13}$ Luigi De Gennaro, ${ }^{14,15}$ Fang Han, ${ }^{16}$ Yuichi Inoue, ${ }^{17,18}$ Kentaro Matsui, ${ }^{19,20}$ Damien Leger, ${ }^{21,22}$ Ana Suely Cunha, ${ }^{23}$ Ilona Merikanto, ${ }^{24}$ Sergio Mota-Rolim, ${ }^{25,26}$ Michael Nadorff, ${ }^{9}$ Giuseppe Plazzi, ${ }^{27,28}$ Jules Schneider, ${ }^{5}$ Mariusz Sieminski, ${ }^{29}$ Yun-Kwok Wing, ${ }^{12}$ Bjørn Bjorvatn (D) 30,31

To cite: Partinen M, Holzinger B, Morin CM, et al. Sleep and daytime problems during the COVID-19 pandemic and effects of coronavirus infection, confinement and financial suffering: a multinational survey using a harmonised questionnaire. BMJ Open 2021;11:e050672. doi:10.1136/ bmjopen-2021-050672

- Prepublication history and additional supplemental material for this paper are available online. To view these files, please visit the journal online (http://dx.doi.org/10.1136/ bmjopen-2021-050672).

Received 03 March 2021 Accepted 17 November 2021

Check for updates

(c) Author(s) (or their employer(s)) 2021. Re-use permitted under CC BY-NC. No commercial re-use. See rights and permissions. Published by BMJ.

For numbered affiliations see end of article.

Correspondence to Dr Markku Partinen; markku.partinen@helsinki.fi

\section{ABSTRACT}

Objectives Sleep is important for human health and well-being. No previous study has assessed whether the COVID-19 pandemic impacts sleep and daytime function across the globe.

Methods This large-scale international survey used a harmonised questionnaire. Fourteen countries participated during the period of May-August 2020. Sleep and daytime problems (poor sleep quality, sleep onset and maintenance problems, nightmares, hypnotic use, fatigue and excessive sleepiness) occurring 'before' and 'during' the pandemic were investigated. In total, 25484 people participated and 22151 (86.9\%) responded to the key parameters and were included. Effects of COVID-19, confinement and financial suffering were considered. In the fully adjusted logistic regression models, results (weighted and stratified by country) were adjusted for gender, age, marital status, educational level, ethnicity, presence of sleep problems before COVID-19 and severity of the COVID-19 pandemic in each country at the time of the survey.

Results The responders were mostly women (64\%) with a mean age 41.8 (SD 15.9) years (median 39, range 18-95). Altogether, 3.0\% reported having had COVID-19; $42.2 \%$ reported having been in confinement; and $55.9 \%$ had suffered financially. All sleep and daytime problems worsened during the pandemic by about $10 \%$ or more. Also, some participants reported improvements in sleep and daytime function. For example, sleep quality worsened in about $20 \%$ of subjects and improved in about $5 \%$. COVID-19 was particularly associated with poor sleep quality, early morning awakening and daytime sleepiness. Confinement was associated with poor sleep quality, problems falling asleep and decreased use of hypnotics. Financial suffering was associated with all sleep and

\section{Strengths and limitations of this study}

- This was a large, multinational internet-based survey on sleep problems, fatigue and sleepiness realised in 14 different countries.

- Harmonised questionnaires were used allowing comparability of results.

- This study gives associations, but one must be cautious in making inferences on causality because there has been no follow-up.

- The results are based on self-report without clinical assessments or sleep recordings.

- A recall bias is possible as concerns any 'before' the pandemic and 'during' the pandemic comparisons.

daytime problems, including nightmares and fatigue, even in the fully adjusted logistic regression models.

Conclusions Sleep problems, fatigue and excessive sleepiness increased significantly worldwide during the first phase of the COVID-19 pandemic. Problems were associated with confinement and especially with financial suffering.

\section{INTRODUCTION}

The COVID-19 pandemic is the deadliest since the 1918 Spanish influenza. The disease exhibits a far higher fatality rate than the seasonal influenza, while also causing both acute and lingering symptoms for many of those afflicted. As of 10 September 2021, there have been more than 223 million confirmed cases and more than 4.6 million 
deaths attributed to COVID-19 worldwide. ${ }^{1}$ Peoples' lives have been affected in many ways.

Sleep is vital for cognitive, emotional and somatic functioning and vice versa. Poor sleep is associated with a multitude of health problems, such as anxiety, depression, suicidal ideation, obesity, cardiovascular diseases, cancer, dementia, increased accident risk and mortality. ${ }^{2}$ Also, sleep problems may increase sick leave and work disability. ${ }^{3}$ Of note, sleep may exhibit a bidirectional relationship with an infectious disease like COVID-19. Poor sleep may compromise in vivo antibody responses to novel antigens, ${ }^{4}$ thus increasing the risk of becoming infected by SARS-CoV-2-virus. ${ }^{5}$ On the other hand, the COVID-19 pandemic may have stressful personal consequences, both practical and emotional, that impair sleep.

Apart from the well-established symptoms of breathing difficulties, fever, loss of smell and taste, COVID-19 has been linked to a range of cognitive and psychiatric symptoms including increased anxiety and depression, ${ }^{6}$ panic attacks, irrational fears, post-traumatic stress, fatigue and sleep disturbances, ${ }^{78}$ and other behavioural factors like 'panic buying' may also be associated with anxiety and poor sleep. ${ }^{9}$ Social isolation, home confinement and loneliness have been associated with increased morbidity ${ }^{6710-12}$ and even increased mortality. ${ }^{10}$

Reduced physical activity and daylight exposure, and a lack of social zeitgebers due to no longer having a fixed work schedule, as well as increased worry may have negatively impacted sleep. ${ }^{13}{ }^{14}$ Nevertheless, lockdown may have had some positive sleep effect. Extrinsically imposed schedules, such as the early morning rush to work, have been replaced by flexible work hours at home. Therefore, sleep duration may have increased and daytime sleepiness decreased for some people,${ }^{1516}$ although sleep quality arising from worries and uncertainties may have remained poor. ${ }^{15}$

The International COVID-19 Sleep Study (ICOSS, https://www2.helsinki.fi/en/projects/icoss) was initiated in March 2020 to improve global understanding of these important relationships. ${ }^{8}$ It includes 14 participating countries across four continents (Asia, Europe, North America and South America). We hypothesised that various sleep-wake problems would increase during, compared with before, the pandemic. Also, we hypothesised that infection with the SARS-CoV-2 virus would be associated with sleep problems, which in turn would strongly correlate with social confinement, familial, workrelated and other psychosocial factors. ${ }^{8}$

\section{Objectives}

The focus of the present study was on the impact of the first wave of the COVID-19 pandemic on sleep and daytime problems. The sleep problems of interest in this study were poor sleep quality, problems of falling asleep (sleep onset problems), problems of maintaining sleep, early morning awakenings, nightmares and use of hypnotics. The sleep-related daytime problems of interest were fatigue and excessive daytime sleepiness. The role of
COVID-19, confinement and financial problems due to the pandemic on these sleep and daytime problems was investigated. Also, we examined country-specific differences in the rates of these sleep and daytime problems.

\section{METHODS}

\section{Survey}

The research protocol and the final standardised survey questionnaire were published previously. ${ }^{8}$ As we aimed to investigate possible changes in the frequency and presentation of various sleep and daytime problems in relation to COVID-19 and confinement, the survey enquired about symptoms and experiences both 'before' and 'during' the pandemic. After gathering data on important sociodemographic variables (age, gender, marital status, etc), the survey incorporated multiple questions on sleep problems using the validated Basic Nordic Sleep Questionnaire. ${ }^{17}$ Scale responses for many of these items such as difficulty falling asleep, problems staying asleep, fatigue, daytime sleepiness and nightmares were 1 , 'never or less frequently than once per month'; 2 , 'less than once per week'; 3, 'on 1-2 days/week'; 4, 'on 3-5 days/week'; and 5 , 'daily or almost daily'. Sleep quality was assessed by the question 'How well have you been sleeping?', with response alternatives 'well', 'rather well', 'neither well nor badly', 'rather badly' and 'badly. ${ }^{8}$ As we wanted to concentrate on clinically meaningful problems, we used mainly a cut-point of 4 (a problem was occurring at least on 3 days/nights per week). The individual cut-points for different questions are given in the results.

Occurrence of COVID-19 was asked as 'Have you had COVID-19?' The response alternatives were 0 , 'no'; 1 , 'yes'; and 2, 'I do not know'. Only those responding yes were defined as having had COVID-19. The participants were also asked, 'Have you been tested positive for corona virus (laboratory test for COVID-19)?': 0, no; 1 , yes; 2, I do not know.

Confinement was asked as 'During the COVID-19 pandemic, have you been restricted to stay at home/ protected/in quarantine?' Response alternatives were 0 , no; 1 , '2 weeks or less'; 2 , '3-4 weeks'; 3 , '5-6 weeks'; 4 , '7-8 weeks"; 5 , 'more than 8 weeks'. The reason for confinement was asked as 'What were your reasons for being restricted to stay at home/ protected/ in quarantine?': 0, 'I have not been restricted to stay at home'; 1 , 'There was a "lockdown" across society'; 2, "I had symptoms or someone in the household had symptoms'; 3, 'Partly because of lockdown, and partly because of symptoms'; 4, 'Due to work regulations'; 5 , 'Due to travelling abroad'. In this study, confinement was dichotomised to 0 , 'no confinement', and 1, 'had been in confinement' for any reason. Financial suffering was based on a single question: 'Has your financial status (economy) suffered from the pandemic?' The response alternatives were 0 , 'not at all'; 1, 'a little'; 2, 'somewhat'; 3, 'much'; 4, 'very much/severely'. We used much or very much/severely to define 'financial suffering' in this study. 
In addition, dates and country of response were recorded. This allowed us to relate the timing of responses to the patterning of pandemic exposure and confinement in each country in accordance with centralised WHO records. ${ }^{1}$

The survey questionnaire ${ }^{8}$ was translated into different languages and administered in 14 countries/areas (Austria, Brazil, Canada, China/Hong Kong, China/Jilin, Finland, France, Italy, Japan, Norway, Poland, Sweden, the UK and the USA) between May and August 2020. To be included in the survey, a minimum of 400 responders with complete answers from any given country had to be available. The most used online platforms for administration of the survey were RedCap and Qualtrics. Potential participants were solicited in each country, for example, by informing about the survey in the university web pages, national newspapers or television, Facebook or Twitter. All responders were anonymous volunteers and aged 18 years or older.

\section{Participants}

In order to be able to adjust results in each country by gender, age (five groups), history of COVID-19 and history of confinement (five periods) and other factors (eg, financial effects, circadian type, etc), we decided in advance, before starting the survey in each country, that the target is at least 400 responders (target $=2 \times 2 \times 2 \times 2 \times 5 \times 5$ ) in each participating country. All participating centres agreed on that. The figure was considered realistic but also large enough to allow tabulations by gender, different age groups, marital status, educational levels and ethnicity. It was not an absolute level, but we used that so that all participating countries would understand why their data might not have been used if there were very few responders. The survey was started in Germany later than in the other participating countries. Therefore, Germany did not have enough responders when we merged the data in the fall 2020, and their data were not included in this study.

A total of 25484 subjects gave their informed consent in the beginning of the survey and participated. Of them, 3333 subjects did not complete the survey in full. After excluding them, 22151 participants $(86.9 \%)$ provided complete data and were included in the analyses.

\section{Patient and public involvement}

There was no patient or public involvement.

\section{Data reduction and analyses}

All survey items and variable names were identical across countries to facilitate merging of data into a single file. This data integration and all statistical computations were conducted using Stata V.15.1. There was no requirement for data imputation for missing data and no replacement of subjects. Shapiro-Wilk test was used for testing of normality. Means and SD were given in the descriptive results if the distributions were normal. It the distributions were not normal, we have also given medians, percentiles and range. For occurrences and rates, we have given the 95\% confidence limits. Multivariate analyses and statistical analyses were conducted by weighting of data by the number of inhabitants in the country/area of interest and by the number of responders in that country. Different countries were used as strata. Observations were used as sampling unit, and non-parametric statistics were applied with proportions and 95\% CIs calculated. Logistic regression analyses and other comparative analyses were used using appropriate weighting for the merged data. We specifically analysed effects of COVID-19 infection (selfreported), confinement for at least 2 weeks and suffering from financial losses because of COVID-19.

\section{RESULTS}

Table 1 presents the demographics of the total study population. The age distribution is shown in table 1. It was skewed to the left (Shapiro-Wilk W 0.955, $\mathrm{p}<0.0001$ ). Ten per cent were aged 18-22 years, and 10\% were aged $65-95$ years. The median age of the responders was 39 years (range 18-95 years, mean 41.8, SD 15.9). In total, $3.0 \%(\mathrm{n}=739)$ reported having had COVID-19. Of these respondents, $404(54.7 \%)$ had been tested positive and $50(6.8 \%)$ did not know if they had been tested positive or not. Altogether, $42.2 \%$ reported having been restricted to their home (in confinement) during the pandemic, and $55.9 \%$ reported having suffered financially due to the pandemic (table 1). Table 1 also presents information on the number of participants from each country.

\section{Sleep and daytime problems before and during COVID-19}

Occurrence of sleep and daytime problems for all participants from the 14 countries is summarised in figure 1 and table 2. The data clearly show that all sleep and daytime problems increased during relative to before the COVID-19 pandemic. In fact, the prevalence of most of the problems (poor sleep quality, sleep onset problems, sleep maintenance problems, fatigue, excessive sleepiness and falling asleep during daytime) increased by about $10 \%$ or more. The smallest percentage increase was in hypnotic use, but even such use increased dramatically from $7.8 \%$ to $12.2 \%$ during the pandemic (figure 1 and table 2).

\section{Worsening and improvement of sleep and daytime problems}

Table 2 provides data on the percentage of participants who reported having improved, unchanged or worsened sleep and daytime problems over the time course of the pandemic. Whereas most participants reported that their sleep and daytime problems were unchanged from before to during the pandemic, sleep quality worsened in about $20 \%$ and improved in about $5 \%$ of participants. Results also differed depending on the variable of interest. For example, $6.9 \%$ reported an increase in hypnotic use and a $20.8 \%$ worsening of sleep quality during the pandemic (table 2). 
Table 1 Characteristics of the study population

$\%(\mathrm{~N}=25454)$

\begin{tabular}{ll}
\hline $\begin{array}{l}\text { Gender }(n=24353) \\
\text { Male }\end{array}$ & $33.9(8257)$ \\
Female & $66.0(16081)$ \\
\hline Other & $0.1(15)$ \\
Age (years) $(n=24218)$ & \\
$<25$ & $14.9(3613)$ \\
\hline $25-34$ & $25.4(6160)$ \\
\hline $35-44$ & $18.7(4525)$ \\
$45-54$ & $16.6(4018)$ \\
$55-64$ & $13.4(3253)$ \\
$65+$ & $10.9(2649)$ \\
\hline
\end{tabular}

Marital status $(n=24264)$

\begin{tabular}{|c|c|}
\hline Single & $36.3(8814)$ \\
\hline Cohabiting & $56.0(13578)$ \\
\hline Divorced/separated & $6.1(1467)$ \\
\hline Widowed & $1.7(405)$ \\
\hline \multicolumn{2}{|l|}{ Education $(n=24069)$} \\
\hline Below university & $73.2(17619)$ \\
\hline College/university & $26.8(6450)$ \\
\hline \multicolumn{2}{|l|}{ Ethnicity (n=23935) } \\
\hline Caucasian/white & $44.2(10588)$ \\
\hline Asian & $40.2(9632)$ \\
\hline Other & $15.5(3715)$ \\
\hline \multicolumn{2}{|l|}{ COVID-19 $(n=24497)$} \\
\hline No & $79.5(19464)$ \\
\hline Yes & $3.0(739)$ \\
\hline Do not know & $17.5(4294)$ \\
\hline \multicolumn{2}{|l|}{ Confinement $(n=23072)$} \\
\hline No & $57.8(13339)$ \\
\hline Two weeks or less & $8.6(1987)$ \\
\hline $3-4$ weeks & $5.1(1187)$ \\
\hline $5-6$ weeks & $4.0(926)$ \\
\hline 7-8 weeks & $5.4(1240)$ \\
\hline More than 8 weeks & $19.0(4393)$ \\
\hline \multicolumn{2}{|c|}{ Financial suffering $(n=24324)$} \\
\hline Not at all & $44.1(10718)$ \\
\hline A little & $28.4(6907)$ \\
\hline Somewhat & $15.1(3662)$ \\
\hline Much & $8.4(2037)$ \\
\hline Very much/severely & $4.1(1000)$ \\
\hline \multicolumn{2}{|l|}{ Country $(n=25484)$} \\
\hline Austria & $3.3(722)$ \\
\hline Brazil & $8.2(1821)$ \\
\hline Canada & $9.4(2080)$ \\
\hline China/Jilin & $4.8(1071)$ \\
\hline
\end{tabular}

Table 1 Continued

\begin{tabular}{lc}
\hline & $\%(\mathbf{N}=\mathbf{2 5 4 5 4})$ \\
\hline China/Hongkong & $8.1(1790)$ \\
\hline Finland & $3.9(869)$ \\
France & $4.9(1089)$ \\
Italy & $6.5(1439)$ \\
Japan & $30.5(6744)$ \\
Norway & $4.8(1060)$ \\
Poland & $2.0(433)$ \\
Sweden & $3.3(733)$ \\
UK & $5.7(1257)$ \\
USA & $4.7(1043)$ \\
\hline
\end{tabular}

Factors associated with the occurrence of sleep and daytime problems

Table 3 presents prevalence data on sleep and daytime problems during the pandemic, segmented by three variables of interest, that is, COVID-19, reports of being in confinement and suffering financially due to the pandemic. Crude (unadjusted) percentages showed that having had COVID-19 was significantly associated with poor sleep quality, early morning awakening, nightmares, hypnotic use, fatigue and excessive sleepiness. In a multiple logistic regression model, all sleep and daytime problems were significantly associated with COVID-19
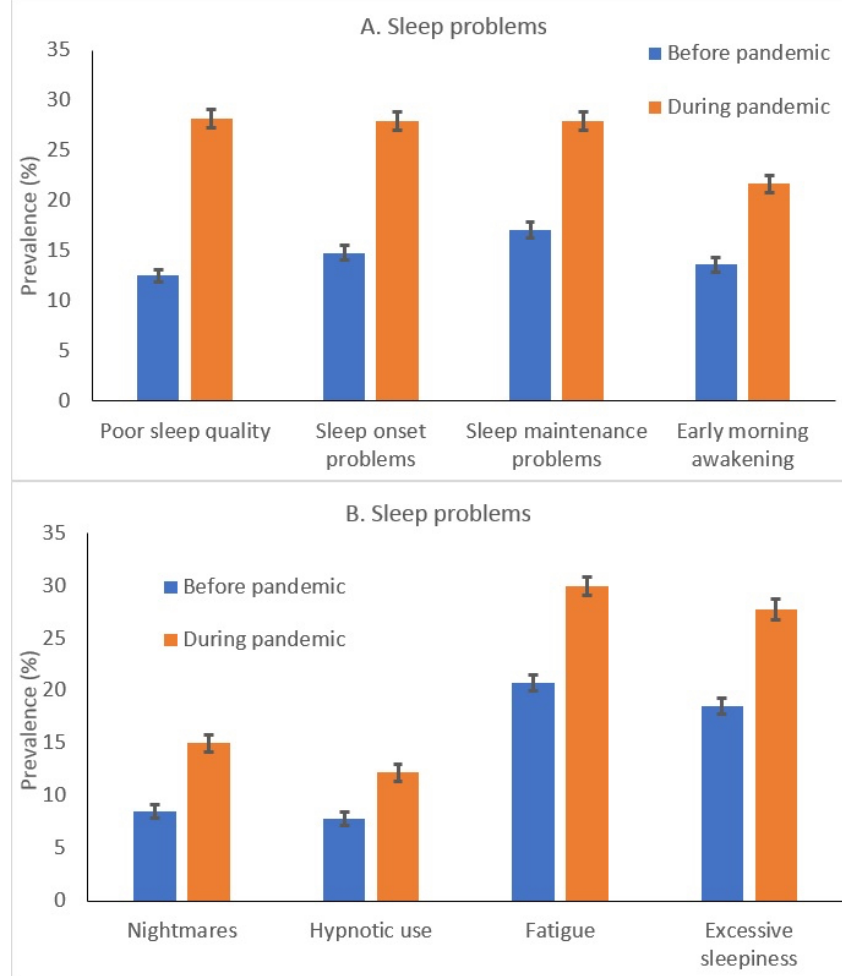

Figure 1 (A,B) Prevalence of all sleep problems before and during the pandemic. There was a statistically significant difference in the prevalence before and during the pandemic for all sleep problems $(p<0.0001)$. Error bars represent SD. 
Table 2 Sleep and daytime problems among all participants in the International COVID-19 Sleep Study study (N=25484).

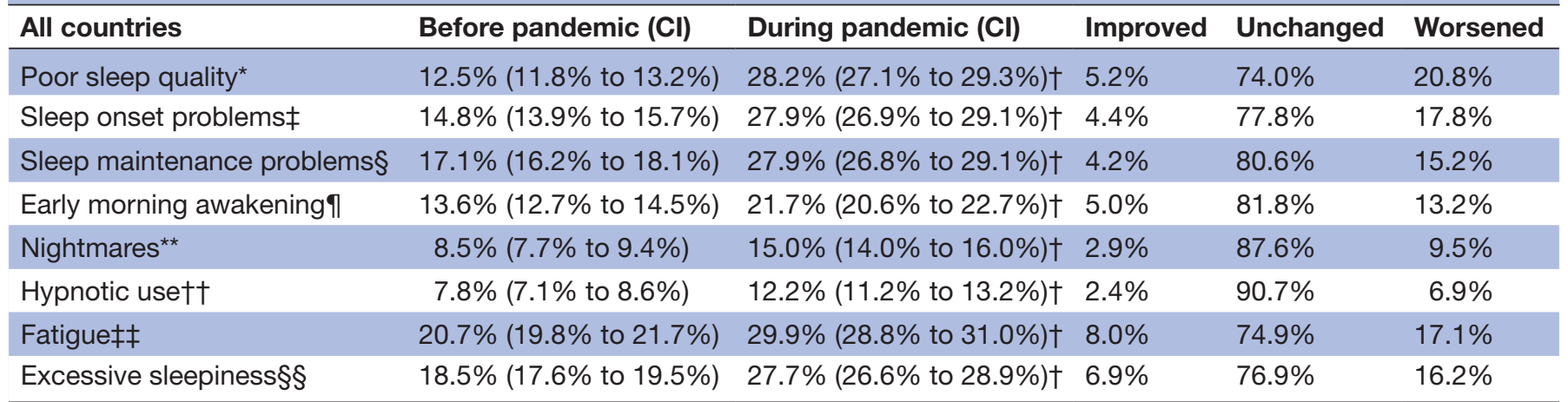

*Proportion reporting sleep quality 'rather badly' or 'badly'.

†Statistically significant difference from before pandemic $(p<0.0001)$.

‡Proportion reporting sleep onset problems 3+ days/week.

§Proportion reporting sleep maintenance problems 3+ day/week.

ๆProportion reporting early morning awakening problems $3+$ days/week.

**Proportion reporting nightmares $3+$ nights/week.

††Hypnotic use 3+ days/week.

‡¥Proportion reporting fatigue $3+$ days/week.

$\S \S P$ roportion reporting excessive daytime sleepiness 3+ days/week.

after adjustment for gender, age, marital status and educational level. However, in the fully adjusted model, with adjustment also for ethnicity, presence of the problem before the pandemic, confinement, financial suffering and the severity of the COVID-19 pandemic in each country at the time of the survey, only poor sleep quality, early morning awakening and sleepiness remained significant (table 3).

Confinement during the pandemic was associated with all the reported sleep and daytime problems in crude as well as in two of the adjusted analyses. However, in the fully adjusted model, confinement was only positively associated with poor sleep quality and sleep onset problems, whereas a negative association with hypnotic use appeared (table 3 ).

Financial suffering during the pandemic was associated with all sleep and daytime problems, both in crude and in all adjusted logistic regression analyses (table 3). The highest OR was seen for hypnotic use, which more than doubled during the pandemic in the fully adjusted model.

\section{Country-specific differences}

Online supplemental tables 1 and 2 present detailed data on sleep and daytime problems reported for each of the 14 countries. Problems increased in all countries during versus before the pandemic, but to differing degrees, and non-significantly for some countries. For instance, poor sleep quality increased in prevalence especially in Canada (nearly four times increase) and the UK (three times higher). Fatigue significantly increased in prevalence in many countries but not in China (Jilin and Hong Kong), France, Italy and Japan. The increase in prevalence of fatigue was particularly pronounced in Sweden and the UK with more than 2.5-fold increase (online supplemental table 2).

\section{DISCUSSION}

Many papers, including meta-analyses, have been published on sleep and sleep disorders related to the pandemic. ${ }^{6} 1819$ However, to our knowledge, this international collaborative study is the first to compare the occurrence of sleep and daytime problems during the pandemic in different countries and continents using harmonised data. ${ }^{8}$

The results of the ICOSS project show that sleep problems increased in prevalence in all countries. More than $20 \%$ of participants reported a worsening of sleep quality. Other sleep-related issues, such as problems with sleep onset and maintenance, nightmares and hypnotic use all increased significantly during the pandemic compared with the situation before.

Interestingly, there were some participants who reported improvements in sleep during the pandemic. This was reflected also in use of hypnotics. While around $6 \%$ used more hypnotics than before the pandemic, around 2\% decreased their hypnotics use. During the pandemic, people in many countries had at least some constraints. Many were forced or recommended to stay at home, and many have been working at home. The resulting worktime flexibility may have helped some people to improve their sleep during the pandemic. This is seen also in decrease of social jetlag during the pandemic. ${ }^{20}$ The prevalence of fatigue and excessive sleepiness increased by about $10 \%$ during the pandemic, but a minority of participants reported improved daytime function (eg, less fatigue and daytime sleepiness).

The COVID-19 pandemic has changed people's lives dramatically. Gatherings of more than a few individuals have been severely limited across public places, at the same time as schools and universities have been closed. Many have had to undergo quarantine or have had to live 


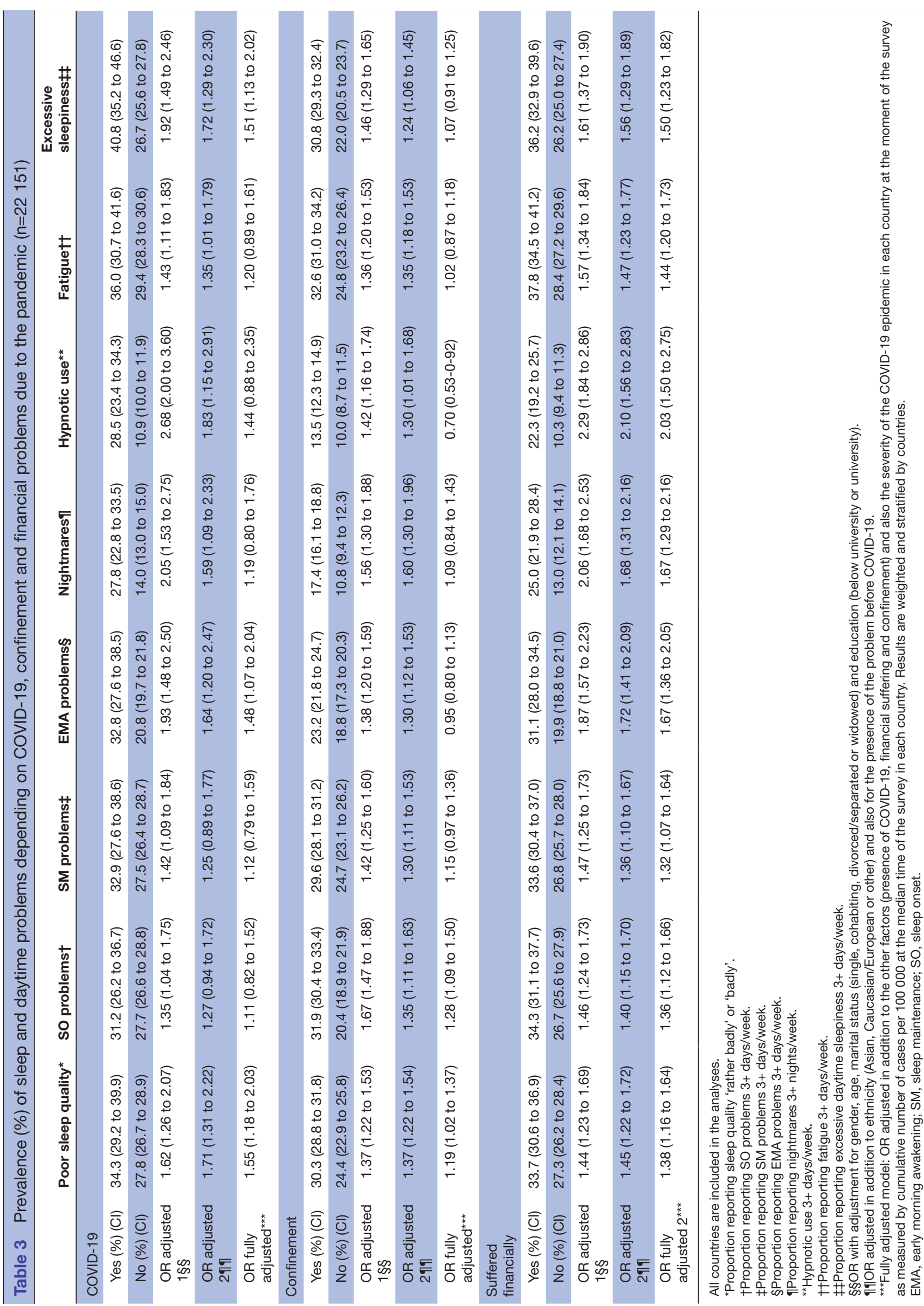


in isolation, in many cases for extended periods of time. In line with our findings of an increase in several sleeprelated problems, other studies have been published in different countries on how people's sleep-wake rhythm has changed during the pandemic. ${ }^{72} 22$ For instance, an Italian study found that more than $50 \%$ of the 6519 participants reported poor sleep quality, with risk factors including female gender, knowing a person who died from COVID-19, changing sleeping habits and a high stress level. ${ }^{22}$ Another reason why the lockdown affected sleep in such drastic ways, apart from isolation, may have been the increasing use of cell phones, laptops and watching TV during lockdown. There have been studies demonstrating that increased screen exposure exacerbates sleep disturbances during lockdown. ${ }^{16}{ }^{23}$ Dutta et al observed that social jet lag and sleep debt were significantly less during than before lockdown in India. ${ }^{23}$ Also, some other studies have shown that sleep may, in fact, be better during the pandemic, in line with our results. ${ }^{162024}$

Anxiety and other psychological effects of confinement were reported previously. ${ }^{725}$ In our survey, confinement was associated especially with problems of falling asleep and poor sleep quality. Interestingly, confinement was negatively associated with hypnotic use, which may be related to the findings that some participants were sleeping well during confinement. Use of hypnotics varied by country. This may reflect different countryspecific attitudes towards use of hypnotics. The effects of confinement are closely related to effects of loneliness, which is related to COVID-19 and to increased mental and physical morbidity. ${ }^{26} 27$

The prevalence of nightmares increased significantly in most countries. This increase may be related to increased levels of stress, anxiety, depression, post-traumatic stress disorder $^{21}$ or even suicidal ideation ${ }^{28}$ during the lockdown caused by the pandemic. Furthermore, nightmares and acting out in sleep are associated with rapid eye movement (REM) sleep behaviour disorder, which in turn is a known risk factor of alpha-synucleinopathies. ${ }^{29}$

In our survey, COVID-19 was associated with poor sleep quality, problems of waking up too early in the morning and daytime sleepiness. The worsening effect of infection on sleepiness may be biological. In a meta-analysis of ten studies, 53\% of patients with COVID-19 had olfactory dysfunction and $44 \%$ had gustatory dysfunction. ${ }^{30}$ Some dysfunction may be long-lasting. ${ }^{31}$ The olfactory bulbs are in contact with the midbrain, including the sleep-wake regulating orexin cell groups. ${ }^{32}$ By retrograde axonal transport, this pathway allows viruses to directly reach the brain and possibly alter brain functions, including sleep and wake regulation. ${ }^{32}$ Our study demonstrates the association between COVID-19, fatigue and sleepiness. This needs to be investigated in more detail in the future. We cannot make strong inferences now because of the cross-sectional nature of our survey. We asked about the symptoms before and during. So, we know about the change related to COVID-19, but we cannot say much about the consequences of a possible
COVID-19. There is increasing evidence of long-lasting symptoms of 'post-COVID syndrome'or 'long COVID' syndrome that may be more prevalent in patients who complain of poor sleep quality. ${ }^{5}$ In addition to respiratory and cardiovascular symptoms, some common complaints among such patients are fatigue, poor sleep quality, postexertional malaise, cognitive disorders and symptoms of orthostatic intolerance-resembling symptoms of myalgic encephalopathy/chronic fatigue syndrome. ${ }^{33-36}$

We found more sleep and daytime problems in countries where the pandemic was escalating at the time of the survey than in countries where the situation was stable. As suggested by the cumulative number of COVID-19confirmed deaths, during spring, the situation was bad in Italy, Spain and France, while during the survey, the worst situations were in Brazil, the USA and Sweden. Use of hypnotics increased especially in Brazil and the USA. Complaints of fatigue, daytime sleepiness and falling asleep at daytime increased clearly in the UK and USA. Sleep problems increased somewhat less in other countries except in China/Jilin, France and Italy, where no significant increase was observed for some of the complaints. Interestingly, the pandemic started in the Wuhan area in China, and in Europe, the pandemic started in the spring 2020, especially in areas with many tourists, such as Austria, Italy, France and Spain. In June and July 2020, new cases continued to rise sharply in Brazil and in the USA as the first wave levelled off in China, Japan and in most European countries.

There are several possible confounders for differences in occurrence of sleep problems including ethnic, cultural and meteorological differences. The ethnic, social and financial differences were included in the logistic regression models. Furthermore, profession and working status during the pandemic may have an effect. We included educational level in the models. However, professions and working status were not included in this study. Also, we have not included effects of seasons. The survey was conducted in May-August 2020, when we had late spring-early summer in the Northern hemisphere and late fall-early winter in the Southern hemisphere (Brazil). The days were very long in the North (Finland, Norway, Sweden and Canada). We cannot make strong inferences, but the situation and occurrences of sleep problems varied between Finland, Norway and Sweden. The differences were most probably due more to political, social and psychological differences between these countries. In Finland and Norway, strict restrictions were used, while there were very few restrictions in Sweden.

The strongest factor associated with all types of sleep and daytime problems was the presence of financial suffering related to COVID-19. The effects of financial suffering may probably be explained by stress and other psychological effects. All these effects influence sleep, autonomic nervous system and immunity. Higher educational level is known to associate with better financial situation. Recent studies have shown also that occurrence 
of COVID-19 is associated with lower educational level, unemployment and low income. ${ }^{37}$

\section{Strengths}

Our survey has multiple strengths. It is based on a harmonised questionnaire with validated questions that were translated into different languages. Prior to our effort, the occurrence of sleep problems has been studied in many countries, but the questions have not been identical, making comparisons between countries difficult. In the first phase of our ICOSS collaboration, we collected data from 14 participating countries using a standardised protocol and a harmonised questionnaire. This allows us to make inferences about worldwide effects of COVID-19, confinement and financial status in different countries.

\section{Limitations}

Our study has several limitations. Recall bias is possible as we asked participants to report on their sleep and daytime problems both before and during the COVID-19 pandemic. The experience of spring 2020 has probably been memorable for most people, but it is possible that some people did not remember how they were sleeping before spring 2020. Even though we tried to be clear in the instructions, some people may have compared before with 'now' rather than during. We know the epidemiological situation in each country now of responding. As we have discussed, and as our results show, the increase in most sleep problems was greatest in countries where the incidence of new cases was increasing at the time of survey (eg, Brazil, the UK and the USA) compared with countries where the situation was more stable at the time of the survey (China/ Jilin, France and Italy). As the situation had been much worse in the latter countries than in, for example, Norway and Finland, it is possible that subjects were thinking of the situation at the time of the response date rather than during the pandemic and confinement.

Some participants with sleep and wake problems may have exaggerated their symptoms during COVID-19. As the survey was done online, people without access to internet and possibly a greater proportion of elderly people would have been unable to participate. This limits generalisation of our results. However, given the observed consistency across countries, we are confident that our results reflect the situation before and during the pandemic. Furthermore, we had young and old responders from both genders and from different ethnic backgrounds, suggesting that our results are representative for a large proportion of citizens in the participating countries.

One important limitation of our study is that the information on having had COVID-19 was based on subjective reports. Mild diseases may have occurred without reporting them. Also, many subjects did not know whether they had been tested positive or not. Therefore, we included on the category of COVID-19 only those who knew that they have had the disease. The study was conducted around the time of the first wave and access to formal testing was limited in spring and summer 2020.

Another limitation is that financial suffering was based on a single question. As this was an anonymous survey, we could not use any objective data. There are important socioeconomic differences between different countries. There are limitations to using a single item to appraise such an important variable. However, there were two reasons that led the study group to decide on this item. First, it was impractical to ask a series of more detailed questions in the context of the survey. Second, and related to the first, we felt it would be very challenging to equate income levels across 14 countries to construct a reliable index of financial hardship. By asking people if they experienced suffering to financial status, we felt we had a reasonable proxy of changes in personal financial circumstances.

The frame of sampling and also sample sizes varied by country and relative to the population of the country. There were also ethnic differences. For these reasons, we have used weighting, but even then, one must be cautious in making strong inferences. The most striking differences were found between the USA and other countries. The responders in the USA were younger and most responders were men, while in other countries, most responders were women.

\section{What needs to be studied?}

Many unanswered questions remain. We have listed our main hypotheses in an earlier publication. ${ }^{8}$ More detailed understanding of the nature of sleep and daytime function during the pandemic is needed. What are the effects of anxiety and depression on sleep and daytime problems? How can we explain the increase of nightmares during the pandemic? The increase of fatigue and daytime sleepiness needs to be studied in more depth. Effects of circadian types ${ }^{38}$ and the relationship between sleep apnoea and COVID-19 must be studied further as well as effects of different factors on symptoms of restless legs and symptoms of REM sleep-behaviour disorder. We would need more subjects having had COVID-19 in order to have better information on the effects of the viral infection and effects of the pandemic situation with restrictions and limitation to 'normal' life. Finally, effects of socioeconomic factors should be studied in more depth in the future.

\section{Clinical implications}

This was an anonymous survey. Therefore, we cannot make any direct inferences on causality. Some of the clinical implications are that also social, economic and psychological factors are important when we treat people during the pandemic. Our results implicate that sleep and daytime problems may be caused by a coronavirus infection, but also by some other traumatic factors that are related to the pandemic, such as confinement and financial suffering. 


\section{CONCLUSIONS}

Sleep problems, fatigue and daytime sleepiness increased significantly worldwide during the first phase of the COVID-19 pandemic. As sleep and health share a bidirectional relationship, such problems were significantly associated with COVID-19, but also with confinement and especially with financial suffering. On a global level, the social and psychological effects seem to play a more important role than the biological effects of the COVID-19 as a disease on these sleep and daytime problems.

\section{Author affiliations}

${ }^{1}$ Department of Clinical Neurosciences, University of Helsinki Clinicum Unit, Helsinki, Finland

${ }^{2}$ Helsinki Sleep Clinic, Terveystalo Healthcare Services, Helsinki, Finland ${ }^{3}$ Institute for Dream and Consciousness Research, Medical University of Vienna, Wien, Austria

${ }^{4}$ Centre d'étude des troubles du sommeil, Centre de recherche CERVO/Brain Research Center, Université Laval École de psychologie, Quebec, Quebec, Canada

${ }^{5}$ Sleep and Circadian Neuroscience Institute, Nuffield Department of Clinical Neurosciences, University of Oxford, Oxford, UK

${ }^{6}$ Department of Anesthesiology and Pain Medicine, University Health Network, Toronto, Ontario, Canada

${ }^{7}$ Sleep Medicine Center, Charite University Hospital Berlin, Berlin, Germany ${ }^{8}$ Department of Neuroscience, Sleep Science (BMC), Uppsala University, Uppsala, Sweden

${ }^{9}$ Department of Psychology, Mississippi State University, Mississippi State, Mississippi, USA

${ }^{10}$ Department of Medical Sciences, Uppsala University, Uppsala, Sweden

${ }^{11}$ Department of Medicine, Division of Endocrinology, Metabolism, and Molecular Medicine, Northwestern University Feinberg School of Medicine, Chicago, Illinois, USA

${ }^{12}$ Li Chiu Kong Family Sleep Assessment Unit, Department of Psychiatry, The Chinese University of Hong Kong, Hong Kong, Hong Kong

${ }^{13}$ National Reference Center for Narcolepsy, Sleep and Wake Unit, Department of Neurology, Gui-de-Chauliac Hospital, University Hospital Centre Montpellier, Montpellier, France

${ }^{14}$ Department of Psychology, Sapienza University of Rome, Roma, Lazio, Italy

${ }^{15}$ IRCCS Fondazione Santa Lucia, Roma, Italy

${ }^{16}$ Department of Pulmonary Medicine, Peking University People's Hospital, Beijing, China

${ }^{17}$ Department of Somnology, Tokyo Medical University, Shinjuku-ku, Japan

${ }^{18}$ Neuropsychiatric Research Institute, Japan Somnology Center, Tokyo, Japan

${ }^{19}$ Department of Clinical Laboratory and Department of Sleep-Wake Disorders, National Center of Neurology and Psychiatry National Institute of Mental Health, Kodaira, Japan

${ }^{20}$ Department of Psychiatry, Tokyo Women's Medical University, Shinjuku-ku, Japan

${ }^{21}$ Sleep and Vigilance Center, Hopital Hotel-Dieu de Paris, Paris, France

${ }^{22}$ VIFASOM (EA 7331 Vigilance Fatigue Sommeil et Santé Publique), Universite de Paris, Paris, France

${ }^{23}$ Production Engineering Department, Federal University of Rio Grande do Norte, Natal, Brazil

${ }^{24}$ Research Programs Unit, Faculty of Medicine, University of Helsinki, Helsinki,

Finland

${ }^{25}$ Brain Institute, Onofre Lopes University Hospital, Petropolis, Brazil

${ }^{26}$ Physiology and Behavior Department, Federal University of Rio Grande do Norte,

Natal, Brazil

${ }^{27}$ IRCCS Istituto Delle Scienze Neurologiche di Bologna, Bologna, Italy

${ }^{28}$ Department of Biomedical, Metabolic and Neural Sciences, University of Modena and Reggio Emilia, Modena, Italy

${ }^{29}$ Department of Emergency Medicine, Medical University of Gdansk, Gdansk, Poland

${ }^{30}$ Department of Global Public Health and Primary Care, University of Bergen, Bergen, Norway

${ }^{31}$ Norwegian Competence Center for Sleep Disorders, Haukeland University Hospital, Bergen, Norway

\section{Twitter Markku Partinen @MarkkuPartinen}

Contributors Members of the core group (BB, FC, CE, BH, CMM, MP and TP) contributed in all phases of the study (conception and design, data collection, data analysis and interpretation, drafting the article, critical revision of the article and final approval of the version to be published). YD and IM contributed in the design of the survey, data collection, data analysis and interpretation, critical revision of the article and final approval of the version to be published. CB, CJB, JC, RNYC, LDG, $\mathrm{FH}, \mathrm{YI}, \mathrm{KM}, \mathrm{DL}, \mathrm{ASC}, \mathrm{SM}-\mathrm{R}, \mathrm{MN}, \mathrm{GP}, \mathrm{JS}, \mathrm{MS}$ and Y-KW contributed in data collection, critical revision of the article and final approval of the version to be published. MP is responsible for the overall content as the guarantor.

Funding The authors have not declared a specific grant for this research from any funding agency in the public, commercial or not-for-profit sectors.

Competing interests FC reports grants from Ontario Ministry of Health Innovation Grant and University Health Network Foundation, and personal fees from Masimo Inc and Takeda Pharma, outside the submitted work. In addition, University Health Network has a patent STOP-Bang questionnaire pending. YI reports personal fees and other from Astellas Pharma, personal fees from Eisai, other from Idorsia Pharmaceuticals Japan, grants from Koike Medical, personal fees from Otsuka Pharmaceutical and grants from Philips Japan outside the submitted work; DL reports grants from grants from Philips (Netherlands), Vanda (USA), Sanofi, Vitalaire International, Merck, and Janssen, Jazz and TYTHM outside the submitted work; KM reports personal fees from Eisai, Meiji Seika Pharma, Mochida, MSD, Otsuka Pharmaceutical and Yoshitomi Pharmaceutical, outside the submitted work; MP reports personal fees and other from Bioprojet, other from Jazz Pharmaceuticals, personal fees from UCB-Pharma, GSK, Takeda and Orion, personal fees and other from MSD and Umecrine, outside the submitted work. MP reports personal fees and other from Bioprojet, other from Jazz Pharmaceuticals, personal fees from UCBPharma, GSK, Takeda and Orion, personal fees and other from MSD and Umecrine, outside the submitted work; TP reports personal fees from Jazz Pharmaceuticals, personal fees from Bayer Healthcare, Neuwirth and Löwenstein Medical, outside the submitted work, and Shareholder of The Siestagroup GmbH, Advanced Sleep Research GmbH, Nukute; GP reports personal fees from UCB-Pharma, Jazz pharmaceuticals, Bioprojet,Idorsia, Takeda, outside the submitted work; Y-KW reports grants from Research Grant Council General Research Fund, grants from Health and Medical Research Fund, personal fees from Eisai Inc, personal fees from Eisai Co., Ltd, other from Lundbeck HK Limited, outside the submitted work.

Patient consent for publication Not applicable.

Ethics approval The anonymous online survey made reidentification of the respondents impossible. All countries obtained approval from their internal reviews board before starting the survey. The ethical diary numbers are Canada REB\#205540, China (Hong Kong) CREC2020.277, France 2218275, Germany EA1/161/20, Italy 0000836, Japan 194, UK R70002/RE001 and USA IRB-20-257. Due to the anonymous nature of survey collection, the regional ethical board did not require an ethical evaluation or approval, and formal ethical committee permissions were exempted in the rest of the participating countries: Austria, Brazil, China (Jilin), Finland, Norway, Poland and Sweden in keeping with the national research governance and regulations. Informed consent was obtained, and the procedures followed all principles that are stated by the Declaration of Helsinki.

Provenance and peer review Not commissioned; externally peer reviewed.

Data availability statement Data are available upon reasonable request.

Supplemental material This content has been supplied by the author(s). It has not been vetted by BMJ Publishing Group Limited (BMJ) and may not have been peer-reviewed. Any opinions or recommendations discussed are solely those of the author(s) and are not endorsed by BMJ. BMJ disclaims all liability and responsibility arising from any reliance placed on the content. Where the content includes any translated material, BMJ does not warrant the accuracy and reliability of the translations (including but not limited to local regulations, clinical guidelines, terminology, drug names and drug dosages), and is not responsible for any error and/or omissions arising from translation and adaptation or otherwise.

Open access This is an open access article distributed in accordance with the Creative Commons Attribution Non Commercial (CC BY-NC 4.0) license, which permits others to distribute, remix, adapt, build upon this work non-commercially, and license their derivative works on different terms, provided the original work is properly cited, appropriate credit is given, any changes made indicated, and the use is non-commercial. See: http://creativecommons.org/licenses/by-nc/4.0/.

ORCID iDs

Markku Partinen http://orcid.org/0000-0002-8182-9368 
Frances Chung http://orcid.org/0000-0001-9576-3606

Thomas Penzel http://orcid.org/0000-0002-4304-0112

Bjørn Bjorvatn http://orcid.org/0000-0001-7051-745X

\section{REFERENCES}

1 WHO COVID-19 Dashboard. Who coronavirus disease (COVID-19) Dashboard, 2021. Available: https://covid19whoint/ [Accessed 19th Sep 2021]

2 Kecklund G, Axelsson J. Health consequences of shift work and insufficient sleep. BMJ 2016;355:i5210.

3 Sivertsen B, Øverland S, Bjorvatn B, et al. Does insomnia predict sick leave? the Hordaland health study. J Psychosom Res 2009;66:67-74

4 Besedovsky L, Lange T, Born J. Sleep and immune function. Pflugers Arch 2012;463:121-37.

5 Zhang J, Xu D, Xie B, et al. Poor-sleep is associated with slow recovery from lymphopenia and an increased need for ICU care in hospitalized patients with COVID-19: a retrospective cohort study. Brain Behav Immun 2020;88:50-8.

6 Rogers JP, Chesney E, Oliver D, et al. Psychiatric and neuropsychiatric presentations associated with severe coronavirus infections: a systematic review and meta-analysis with comparison to the COVID-19 pandemic. Lancet Psychiatry 2020;7:611-27.

7 Huang Y, Zhao N. Generalized anxiety disorder, depressive symptoms and sleep quality during COVID-19 outbreak in China: a web-based cross-sectional survey. Psychiatry Res 2020;288:112954.

8 Partinen M, Bjorvatn B, Holzinger B, et al. Sleep and circadian problems during the coronavirus disease 2019 (COVID-19) pandemic: the International COVID-19 sleep study (ICOSS). J Sleep Res 2021;30:e13206.

9 Yuen KF, Wang X, Ma F, et al. The psychological causes of panic buying following a health crisis. Int $J$ Environ Res Public Health 2020;17:3513.

10 Uchino BN. Social support and health: a review of physiologica processes potentially underlying links to disease outcomes. J Behav Med 2006;29:377-87.

11 Beck F, Léger D, Fressard L, et al. Covid-19 health crisis and lockdown associated with high level of sleep complaints and hypnotic uptake at the population level. J Sleep Res 2021;30:e13119.

12 Ammar A, Mueller P, Trabelsi K, et al. Psychological consequences of COVID-19 home confinement: the ECLB-COVID19 multicenter study. PLoS One 2020;15:e0240204

13 Cellini N, Canale N, Mioni G, et al. Changes in sleep pattern, sense of time and digital media use during COVID-19 lockdown in Italy. $J$ Sleep Res 2020;29:e13074.

14 Altena E, Baglioni C, Espie CA, et al. Dealing with sleep problems during home confinement due to the COVID-19 outbreak: practical recommendations from a task force of the European CBT-I Academy. J Sleep Res 2020;29:e13052.

15 Blume C, Schmidt MH, Cajochen C. Effects of the COVID-19 lockdown on human sleep and rest-activity rhythms. Curr Biol 2020;30:R795-7.

16 Wright KP, Linton SK, Withrow D, et al. Sleep in university students prior to and during COVID-19 Stay-at-Home orders. Curr Biol 2020;30:R797-8.

17 Partinen M, Gislason T. Basic Nordic sleep questionnaire (BNSQ): a quantitated measure of subjective sleep complaints. J Sleep Res 1995;4:150-5.

18 Pappa S, Ntella V, Giannakas T, et al. Prevalence of depression, anxiety, and insomnia among healthcare workers during the COVID-19 pandemic: a systematic review and meta-analysis. Brain Behav Immun 2020;88:901-7.
19 Salari N, Khazaie H, Hosseinian-Far A, et al. The prevalence of sleep disturbances among physicians and nurses facing the COVID-19 patients: a systematic review and meta-analysis. Global Health 2020;16:92

20 Korman M, Tkachev V, Reis C, et al. COVID-19-mandated social restrictions unveil the impact of social time pressure on sleep and body clock. Sci Rep 2020;10:22225.

21 Casagrande M, Favieri F, Tambelli R, et al. The enemy who sealed the world: effects quarantine due to the COVID-19 on sleep quality, anxiety, and psychological distress in the Italian population. Sleep Med 2020;75:12-20.

22 Gualano MR, Lo Moro G, Voglino G, et al. Effects of Covid-19 Lockdown on mental health and sleep disturbances in Italy. Int $J$ Environ Res Public Health 2020;17:4779-13.

23 Dutta K, Mukherjee R, Sen D. Effect of COVID-19 lockdown on sleep behavior and screen exposure time: an observational study among Indian school children. Biol Rhythm Res 2020.

24 Leone MJ, Sigman M, Golombek DA. Effects of lockdown on human sleep and chronotype during the COVID-19 pandemic. Curr Biol 2020;30:R930-1.

25 Liu N, Zhang F, Wei C, et al. Prevalence and predictors of PTSS during COVID-19 outbreak in China hardest-hit areas: gender differences matter. Psychiatry Res 2020;287:112921.

26 Groarke JM, Berry E, Graham-Wisener L, et al. Loneliness in the UK during the COVID-19 pandemic: cross-sectional results from the COVID-19 psychological wellbeing study. PLoS One 2020;15:e0239698.

27 Noone C, McSharry J, Smalle M, et al. Video calls for reducing socia isolation and loneliness in older people: a rapid review. Cochrane Database Syst Rev 2020;5:CD013632.

28 Musse FCC, Castro LdeS, Sousa KMM, et al. Mental violence: the COVID-19 nightmare. Front Psychiatry 2020;11:579289.

29 Postuma RB, Iranzo A, Hu M, et al. Risk and predictors of dementia and parkinsonism in idiopathic REM sleep behaviour disorder: a multicentre study. Brain 2019;142:744-59.

30 Tong JY, Wong A, Zhu D, et al. The prevalence of olfactory and gustatory dysfunction in COVID-19 patients: a systematic review and meta-analysis. Otolaryngol Head Neck Surg 2020;163:3-11.

31 Hopkins C, Surda P, Whitehead E, et al. Early recovery following new onset anosmia during the COVID-19 pandemic - an observational cohort study. J Otolaryngol Head Neck Surg 2020;49:26

32 Tesoriero C, Codita A, Zhang M-D, et al. H1N1 influenza virus induces narcolepsy-like sleep disruption and targets sleepwake regulatory neurons in mice. Proc Natl Acad Sci U S A 2016;113:E368-77.

33 Islam MF, Cotler J, Jason LA. Post-Viral fatigue and COVID-19: lessons from past epidemics. Fatigue: Biomedicine, Health \& Behavior 2020;8:61-9.

34 Newman M. Chronic fatigue syndrome and long covid: moving beyond the controversy. BMJ 2021;373:n1559.

35 Mahase E. Covid-19: What do we know about "long covid"? BMJ 2020;370:m2815

36 Al-Aly Z, Xie Y, Bowe B. High-dimensional characterization of postacute sequelae of COVID-19. Nature 2021;594:259-64.

37 Pieh C, Budimir S, Probst T. The effect of age, gender, income, work, and physical activity on mental health during coronavirus disease (COVID-19) lockdown in Austria. J Psychosom Res 2020;136:110186.

38 Merikanto I, Kortesoja L, Benedict C, et al. Evening-types show highest increase of sleep and mental health problems during the COVID-19 pandemic - Multinational study on 19,267 adults. Sleep 2021:zsab216. 